\title{
On the Singularities of Reissner-Nordström Space-Time
}

\author{
M. Abdel-Megied and Ragab M. Gad \\ Mathematics Department, Faculty of Science \\ Minia University, 61915 El-Minia, Egypt *
}

\begin{abstract}
It is shown that if two Reissner-Nordström space-times, both with the same mass $\mathrm{m}$ and charge e, glued together in the singularities, then the light ray in black hole of the first space-time can go continuously through the singularity into black hole of the second. The behavior of tidal forces near the Reissner-Nordström space-time singularity is examined by considering what happens between two particles falling freely towards the singularity.
\end{abstract}

\section{Introduction}

A well known spherically symmetric solution of the Einstein Maxwell equations is the Reissner-Nordström space-time. It represents a non-rotating black hole with a mass $\mathrm{m}$ and a charge e. The basic properties of this solution are discussed by many authors [6], [16], and [23].

Gad [8] studied the possibility of gluing two Schwarzschild space-times in their singularities. It is shown that if two Schwarzschild space-times, both with the same mass, glued together in the singularities, then the light ray in black hole of the first space-time can go continuously through the singularity into black hole of the second. This study was by well defining of the passage of particles through the singularity. Although such gluing of singular points is, of course, not possible within the theory of differential manifolds, because the differential manifold structure of space-time breaks down at a singularity.

In the last two decades, somome mathematical frameworks have been found to generalize the space-time model and consider it to be a differential space rather than a differential manifold. For instance, a generalized model of space-time has been studied in a series of papers by Gruszczak [13] - [15], and Heller et al [17]. The theory of differential spaces open the possibilities to include singularities of space-time. We use here a special form of differential spaces studied in [10], [11], [12].

The aim of this paper is twofold, one is to study the possibility of gluing two Reissner-Nordström space-times in their singularities, the second is to study the behavior of tidal forces near Reissner-Nordström space-time singularity by examining what happens between two particles falling freely towards the singularity.

In the this work, the Reissner-Nordström solution together with its singularities is consider as d-space [10], [11].

*e-mail:amegied@frcu.eun.eg, ragab2gad@hotmail.com 
Since the theory of differential spaces is not commonly known, we give its basic definition in sec. 2. A brief discussion of the geodesics in the Reissner-Nordström space-time is given in sec. 3. In sec. 4 it is shown that: in the case of radial null geodesics, two Reissner-Nordström space-times can be glued in their singularities. The behavior of the Jacobi vector fields near the Reissner-Nordström singularity are studied in sec. 5 .

\section{2 d-Spaces}

Since the theory of d-spaces, introduced by [10], [11] [12], is not widely known, we will present in this section the necessary basic concepts of $\mathrm{d}$-spaces.

Definition 1: Let $M$ be a topological space. The pair $(M, C)$ is called differential space ( or d-space), and $C$ differential structure, if $C$ is a sheaf of continuous real-valued functions on $M$ which form an algebra (w.r.t. pointwise operation). This definition generalizes Sikorski's definition [22] which requires global functions. In addition, the composition of function $g \in C^{\infty}\left(\mathbf{R}^{\mathbf{n}}\right)$ with $n$ arbitrary functions of $C$ must again be a function of $C$, and $M$ must carry the initial topology of $C$. The latter axiom is one of the reasons, why Sikorski's definition is not able to describe the singularities in general relativity [18]. Therefore, Heller and Sasin proposed [19] to use Mostow spaces [21] which called later structured spaces. These spaces are slightly more special than the d-spaces of definition 1 .

Definition 2: Let $(M, C)$ be a d-space, $x \in M, C_{x}$ the stalk at $x$. A map

$$
V: C_{x} \rightarrow
$$

is called tangent vector to $(M, C)$ in $x$, if for all $n \in N a t u$, all $f_{1}, \ldots, f_{n} \in C_{x}$, and all germs $\alpha$ of $C^{1}\left(\mathbf{R}^{\mathbf{n}}\right)$ at $y:=\left(f_{1}(x), \ldots, f_{n}(x)\right) \in \mathbf{R}^{\mathbf{n}}$, the equation

$$
V\left(\alpha \circ\left(f_{1}(x), \ldots, f_{n}(x)\right)\right)=\Sigma_{i=1}^{n}\left(\partial_{i} \alpha\right) \cdot V\left(f_{i}\right)
$$

holds, provided $\alpha \circ\left(f_{1}(x), \ldots, f_{n}(x)\right) \in C_{x}$. Here $\partial_{i} \alpha$ denotes the partial derivative of $\alpha$ w.r.t. the $i$-th argument.

The vector space of all tangent vectors to $(M, C)$ in $x$ is called tangent space $T_{x} M$.

The dimension of $T_{x} M$ can easily be computed:

We call a subset $F$ of the stalk $C_{x}$ dependent, if there is a germ $g \in F$ and a finite subset $\left\{f_{1}, \ldots, f_{n}\right\} \subseteq F$ not containing $g$ such that

$$
g=\alpha \circ\left(f_{1}, \ldots, f_{n}\right)
$$

holds for some $C^{1}$-functions $\alpha$. Now choose any independent subset $G \subseteq C_{x}$ such that all germs $f \in C_{x}$ are a combination of finitely many elements of $G$, i.e.

$$
f=\alpha \circ\left(f_{1}, \ldots, f_{n}\right)
$$

holds for some $n$, some local $C^{1}$-function $\alpha$ on $\mathbf{R}^{\mathbf{n}}$, and some $f_{1}, \ldots, f_{n} \in G$. Write $G$ as $G=\left\{f_{j}\right\}_{j \in J}$. Then definition 2 shows that $\left\{V_{j}\right\}_{j \in J}$ defined by

$$
V_{j}\left(f_{k}\right)=\delta_{j k}
$$


is a basis of $T_{x} M$.

On d-spaces, differentiation, differential forms and exterior derivative are introduced [10], [11] in a way similar to Sikorski's differential spaces.

The topology of space-times with singularities has been a subject of frequent discussions (e.g. in [3], [9], [19]). Also the definition of a d-space requires an a priory given topology. But definition 2 shows that the initial topology of $C$ cannot be finer than this a priory topology. This leads to the following definition Definition3: Let $(M, \varrho)$ be a topological space, and $F$ a sheaf of local function $M$ defined w.r.t. the topology $\varrho$. A topology $\alpha$ on $M$ that is coarser than $\varrho$, is called a slackening of $F$, if for every $V \in \varrho$ and every $f \in F(V)$, there are $U \in \alpha ; U \supseteq V$, and $g \in F(U)$ such that $f=\left.g\right|_{V}$ holds. It is shown in [10], [11] that there exits a coarsest slackening $\mu$, such that all functions $g \in F(U) ; U \in \mu$ are continuous. This $\mu$ is called initial topology of $(M, C)$ It is not difficult to construct this initial topology explicitly (see [10], [11]).

\section{Geodesics in the Reissner-Nordström Space-Times}

The Reissner-Nordström solution is given by the following metric [16]

$$
d s^{2}=-\left(1-\frac{2 m}{r}+\frac{e^{2}}{r^{2}}\right) d t^{2}+\left(1-\frac{2 m}{r}+\frac{e^{2}}{r^{2}}\right)^{-1} d r^{2}+r^{2}\left(d \theta^{2}+\sin ^{2} \theta d \phi^{2}\right),
$$

where $\mathrm{m}$ represents the gravitational mass and e the electric charge of the body.

The geodesic equations for the metric (2) are given by the following equations

$$
\begin{gathered}
\frac{d u^{1}}{d \tau}+\frac{e^{2}-m r}{r\left(r^{2}-2 m r+e^{2}\right)}\left(u^{1}\right)^{2}-\frac{r^{2}-2 m r+e^{2}}{r}\left(u^{2}\right)^{2}-\frac{r^{2}-2 m r+e^{2}}{r} \sin ^{2} \theta\left(u^{3}\right)^{2}+ \\
\frac{\left(\frac{m}{r^{2}}-\frac{e^{2}}{r^{3}}\right)\left(r^{2}-2 m r+e^{2}\right)}{r^{2}}\left(u^{4}\right)^{2}=0 \\
\frac{d u^{2}}{d \tau}+\frac{2}{r} u^{1} u^{2}-\sin \theta \cos \theta\left(u^{3}\right)^{2}=0 \\
\frac{d u^{3}}{d \tau}+\frac{2}{r} u^{1} u^{3}+2 \cot \theta u^{2} u^{3}=0 \\
\frac{d u^{4}}{d \tau}+\frac{2\left(-m+\frac{e^{2}}{r}\right)}{r^{2}-2 m r+e^{2}} u^{1} u^{4}=0
\end{gathered}
$$

where $\tau$ is an affine parameter, $u^{a}=\frac{d x^{a}}{d \tau}$ and $x^{a}(\tau)$ are the coordinates of a space-time point on the geodesic.

Let the geodesic $\gamma$ be given by $\gamma(\tau)=(r(\tau), \theta(\tau), \phi(\tau), t(\tau))$. Without loss of generality We suppose that $\gamma\left(\tau_{0}\right)=\left(r_{0}, \theta_{0}, \frac{\pi}{2}, t_{0}\right)$ and for all $\tau: \phi=\frac{\pi}{2}$. Then $\frac{d \phi}{d \tau}=u^{3}=0$ and the above equations become

$$
\begin{gathered}
\frac{d u^{1}}{d \tau}+\frac{e^{2}-m r}{r\left(r^{2}-2 m r+e^{2}\right)}\left(u^{1}\right)^{2}-\frac{r^{2}-2 m r+e^{2}}{r}\left(u^{2}\right)^{2}+ \\
\frac{\left(\frac{m}{r^{2}}-\frac{e^{2}}{r^{3}}\right)\left(r^{2}-2 m r+e^{2}\right)}{r^{2}}\left(u^{4}\right)^{2}=0, \\
\frac{d u^{2}}{d \tau}+\frac{2}{r} u^{1} u^{2}=0,
\end{gathered}
$$




$$
\begin{gathered}
\frac{d u^{3}}{d \tau}=0 \\
\frac{d u^{4}}{d \tau}+\frac{2\left(-m+\frac{e^{2}}{r}\right)}{r^{2}-2 m r+e^{2}} u^{1} u^{4}=0 .
\end{gathered}
$$

Integrating equations (8) and (10), we obtain respectively

$$
\begin{gathered}
u^{2}=\frac{c_{2}}{r^{2}}, \\
u^{4}=\frac{c_{1} r^{2}}{r^{2}-2 m r+e^{2}},
\end{gathered}
$$

where the integrating constant $c_{1}$ represents the energy $E$ (at $r \rightarrow \infty$ ) of a test particle and $c_{2}$ the angular momentum $L$ [23].

Substituting (11) and (12) in (2), using the condition $u^{3}=0$, we get

$$
\left(u^{1}\right)^{2}=c_{1}^{2}+\left(k-\frac{c_{2}^{2}}{r^{2}}\right)\left(1-\frac{2 m}{r}+\frac{e^{2}}{r^{2}}\right) .
$$

For timelike geodesics, $k=-1$, equation (13) implies

$$
u^{1}= \pm\left[c_{1}^{2}-\left(1+\frac{c_{2}^{2}}{r^{2}}\right)\left(1-\frac{2 m}{r}+\frac{e^{2}}{r^{2}}\right)\right]^{\frac{1}{2}} .
$$

Similarly, $k=0$ corresponds to null geodesics, thus we have

$$
u^{1}= \pm\left[c_{1}^{2}-\left(\frac{c_{2}^{2}}{r^{2}}\right)\left(1-\frac{2 m}{r}+\frac{e^{2}}{r^{2}}\right)\right]^{\frac{1}{2}} .
$$

The \pm sign is used in equations (14) and (15) since $\frac{d r}{d \tau}>0$ holds at each point on a geodesic where it is outward and $\frac{d r}{d \tau}<0$ where it is inward.

To investigate what happens when the null geodesics approach the singularity $r=$ 0 . Therefore we shall be interested in geodesics on which $r$ decreases as the affine parameter $\tau$ increases, that is, $\frac{d r}{d \tau}<0$. Hence the minus sign will be chosen in equation (14) and (15). Also we restrict ourselves to the region $0<r<r_{-}$(assuming $m>a b s e$ ).

\section{Gluing of Two Reissner-Nordström Space-Times in Their Singularities}

Now we study the gluing of two Reissner-Nordström space-times $M_{1}$ and $M_{2}$ in the singularity, $M_{1}$ and $M_{2}$ being given respectively as follows

$$
\begin{aligned}
& d s_{1}^{2}=-\left(1-\frac{2 m_{1}}{r}+\frac{e_{1}^{2}}{r^{2}}\right) d t^{2}+\left(1-\frac{2 m_{1}}{r}+\frac{e_{1}^{2}}{r^{2}}\right)^{-1} d r^{2}+r^{2}\left(d \theta^{2}+\sin ^{2} \theta d \phi^{2}\right), \\
& d s_{2}^{2}=-\left(1-\frac{2 m_{2}}{r}+\frac{e_{2}^{2}}{r^{2}}\right) d t^{2}+\left(1-\frac{2 m_{2}}{r}+\frac{e_{2}^{2}}{r^{2}}\right)^{-1} d r^{2}+r^{2}\left(d \theta^{2}+\sin ^{2} \theta d \phi^{2}\right) .
\end{aligned}
$$

First we notice that we must have $m_{1}=m_{2}$ and $e_{1}=e_{2}$, if $M_{1} \cup M_{2}$ is a differential space, that is, a solution of Einstein's equation. Although there is not yet a general theory of partial differential equations on differential spaces, Einstein equations can be treated in this special case, where an open dense subset of the space is a differential 
manifold [5]. So $M_{1} \cup M_{2}$ is one single solution with the same integration constants, that is, $m_{1}=m_{2}$ and $e_{1}=e_{2}$.

We consider radial null geodesics with zero angular momentum $\left(c_{2}=L=0\right)$, then it follows from (15), (12) and (11) that

$$
\begin{gathered}
u^{1}= \pm E, \\
u^{4}=E\left(1-\frac{2 m}{r}+\frac{e^{2}}{r^{2}}\right)^{-1}, \\
u^{2}=u^{3}=0 .
\end{gathered}
$$

Dividing (16) by (17), we obtain

$$
\frac{d r}{d t}= \pm\left(1-\frac{2 m}{r}+\frac{e^{2}}{r^{2}}\right)
$$

The minus sign is used in the region $r<r_{-}\left(r_{ \pm}=m \pm \sqrt{m^{2}-e^{2}}\right.$ are the roots of the quadratic $\Delta=r^{2}-2 m r+e^{2}$ ), because in this region $r$ decreases with increasing $\tau$, that is, $\frac{d r}{d t}<0$.

Integrating (19) gives

$$
t=-r_{\star}+\text { constant }
$$

where

$$
r_{\star}=r+m \ln a b s r^{2}-2 m+e^{2}+\frac{2 m^{2}-e^{2}}{\sqrt{m^{2}-e^{2}}} \ln a b s \frac{r-r_{+}}{r-r_{-}} .
$$

Equation (20) must be contrasted with the equation

$$
r= \pm E \tau+\text { textconstant }
$$

which relates $r$ to the proper time $\tau$. The surface $r=r_{+}$is an event horizon in the same sense that $r=2 m$ is an event horizon in Schwarzschild space-time. Also the surface $r=r_{-}$is a horizon [6]. Equation (20) and (21) show that while the radial geodesic crosses the horizon $r=r_{-}$in within finite proper time without ever noticing it, it takes an infinite coordinate time even to arrive at the horizon. This is clear from equation (17): When the geodesic approaches the horizon $r=r_{-}$the tangent in the $t$-direction tends to infinity (see, Fig. (1)).

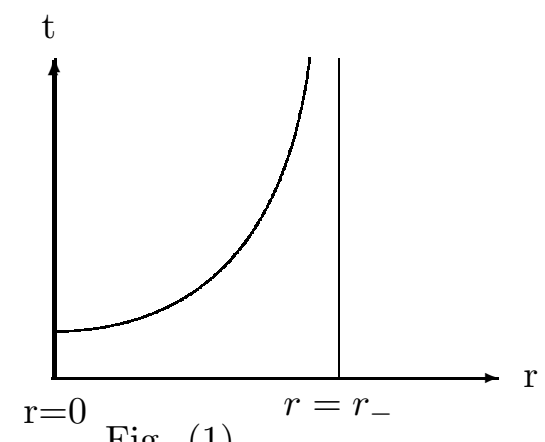

Fig. (1)

At the singularity $r=0$ the tangent component in the $t$-direction tends to zero and in the $r$-direction remains constant. It is shown from the definition of black hole that in the region $r<r_{-}$no light ray can remain at a constant value of $r$ but must move inwards to fall in the singularity $r=0$ and it can never escape to larger values of $r$ to 
communicate with external light ray. This means that any light ray fallen in the black hole can never leave this black hole again.

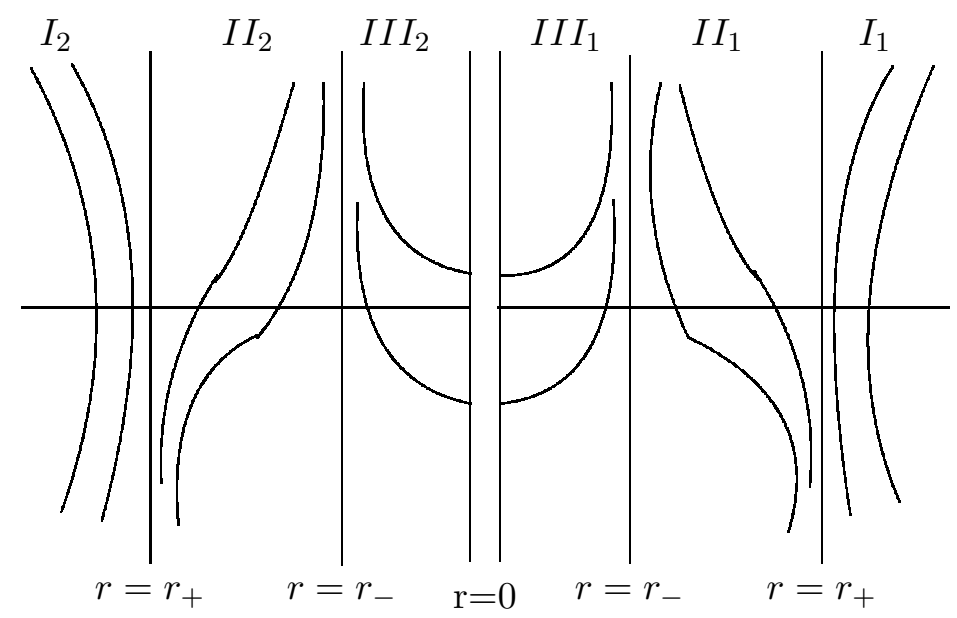

Fig. (2)

Consider two Reissner-Nordström space-times $M_{1}$ and $M_{2}$, both with the same mass and the same charge, and glued together in the singularities.

The question arises: Can the light leave the black hole of $M_{1}$ to fall into the black hole of $M_{2}$ ?

To answer this question, we assume that the two black holes are represented by the two regions $I I I_{1}$ and $I I I_{2}$ (see, Fig. (2)), and we see what happens at $r=0$.

Now in region $I I I_{1}$ the end point of the trajectory may be considered as initial condition $\left(u^{1}=+E, u^{4}=0\right)$ in region $I I I_{2}$ at $r=0$. Then a light ray in region $I I I_{1}$ falling into the singularity can go continuously through the singularity into region $I I I_{2}$ along the world line $\gamma_{2}$ (see Fig. (3) and Fig. (4)).

We considered the radial null geodesics, with zero angular momentum, running into the black hole. Some of them have a well-defined limit at $r=0$ (see equations (16) and (17)). This open the possibility to ask, what happens to geodesics beyond the singularity? and we try gluing two space-times in their singularities. This gluing can be viewed from the mathematical point of view: since the metric coefficients depend only on $r$, we can see that the metric and its first and second derivatives agree on the two sides of the glued two space-times. This satisfy the junction condition for gluing two space-times [20]. 


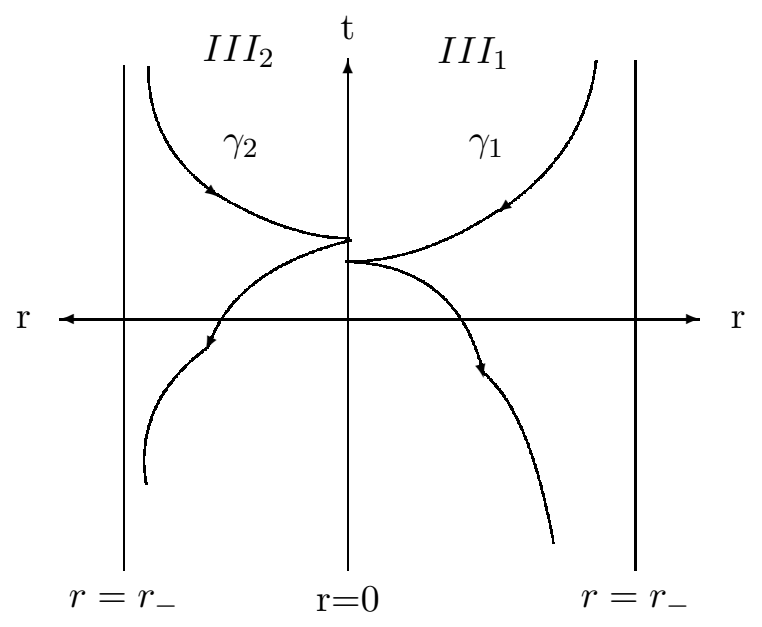

Fig. (3)

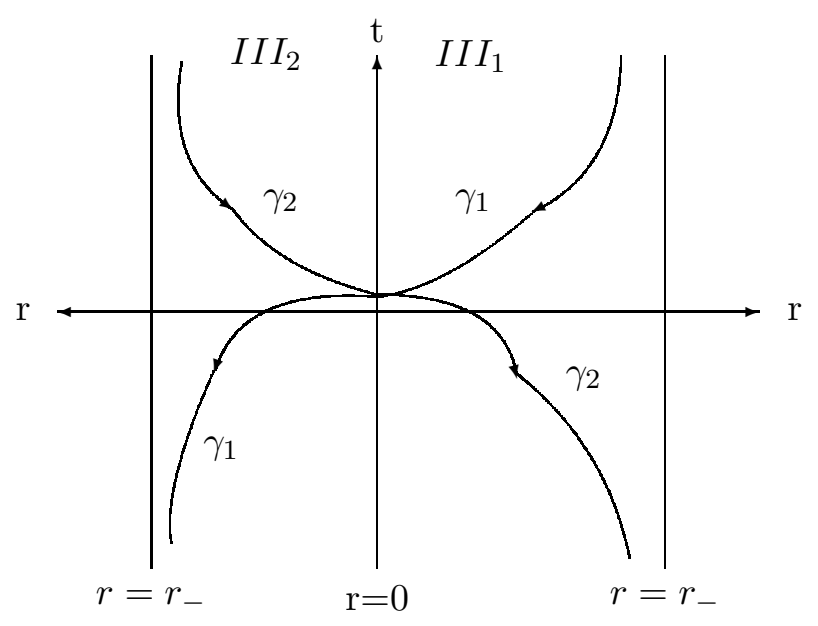

Fig. (4)

\section{Tidal Forces Near the Singularities}

A physical meaning of Riemann curvature tensor is that its components describe tidal forces (relative accelerations) between two particles in free fall. Consider a body falling towards a black hole, then the effect of the gravitational attraction becomes infinite as the singularity is reached. Jacobi vector fields provide the connection between the behavior of nearby particles and curvature, via the equation of geodesic deviation (Jacobi equation)

$$
\frac{D^{2} \eta^{a}}{D \tau^{2}}+R_{b c d}^{a} v^{b} v^{c} \eta^{d}=0
$$

where $v^{a}$ are the components of the tangent vector to geodesic and $\eta^{a}$ are the components of the connecting vector between two neighboring geodesics.

In order to investigate in detail the behavior of Jacobi fields we consider a congruence of timelike geodesics (path of particles) with timelike unit tangent vector $v$ $(g(v, v)=-1)$. We define at some point $q$ on the geodesic $\gamma(\tau)$ dual bases $e_{1}^{a}, e_{2}^{a}, e_{3}^{a}, e_{4}^{a}$ 
and $e_{a}^{1}, e_{a}^{2}, e_{a}^{3}, e_{a}^{4}$ of the tangent space $T_{q} M$ and dual tangent $T_{q}^{\star} M$ respectively in the following way [7] : We choose $e_{4}^{a}$ to be $v^{a}$ and $e_{1}^{a}, e_{2}^{a}, e_{3}^{a}$ as unit spacelike vectors, orthogonal to each other and to $v^{a}$. If we parallelly propagate the basis along the timelike geodesic $\gamma(\tau)$ (that is, $\left.\frac{D}{D \tau} e_{i}^{a}=0, i=1,2,3\right)$, $e_{4}^{a}$ will remain equal $v^{a}$, and $e_{1}^{a}, e_{2}^{a}, e_{3}^{a}$ will remain to orthogonal to $v^{a}$ (see [16] p. 80). The frame $e_{1}^{a}, e_{2}^{a}, e_{3}^{a}, e_{4}^{a}$ is called "parallel transported" (PT) frame. The orthogonal connecting vector, $\eta^{a}$, between two neighboring timelike geodesics may be expressed as $\eta^{a}=\eta^{\alpha} e_{\alpha}^{a}\left(\eta^{4}=e_{\alpha}^{4} \eta^{\alpha}=0\right)$, where Greek indices take the value $1,2,3$.

The Jacobi vector fields $\eta^{a}$ satisfy the following equation

$$
\begin{gathered}
\frac{D \eta^{\alpha}}{D \tau}=\eta_{; a}^{\alpha} v^{a} \\
\frac{D^{2} \eta^{\alpha}}{D \tau^{2}}+\tilde{R}_{b d c}^{a} e_{a}^{\alpha} v^{b} v^{c} e_{\beta}^{d} \eta^{\beta}=0
\end{gathered}
$$

where $\eta^{\alpha}, \alpha=1,2,3$, are the space-like components of the orthogonal connecting vector $\eta^{a}$ connecting two neighboring particles in free fall; $\eta^{4}=0$. The tilde denotes components in the PT frame and the components of the Riemann tensor $\tilde{R}_{b d c}^{a}$ are given by

$$
\tilde{R}_{b d c}^{a}=e_{e}^{a} e_{b}^{f} e_{c}^{g} e_{d}^{h} R_{f g h}^{e} .
$$

From (2) the frame $e_{i}^{a}$ in Reissner-Nordström metric is given by :

$$
\begin{gathered}
e_{1}^{a}=\left(1-\frac{2 m}{r}+\frac{e^{2}}{r^{2}}\right)^{\frac{1}{2}}(1,0,0,0), \\
e_{2}^{a}=r^{-1}(0,1,0,0) \\
e_{3}^{a}=(r \sin \theta)^{-1}(0,0,1,0) \\
e_{4}^{a}=\left(1-\frac{2 m}{r}+\frac{e^{2}}{r^{2}}\right)^{-\frac{1}{2}}(0,0,0,1)
\end{gathered}
$$

The components of $\eta^{\alpha}$ can be written as follows

$$
\eta^{\alpha}=\left(\eta^{1}, \eta^{2}, \eta^{3}\right)=\left(\eta^{r}, \eta^{\theta}, \eta^{\phi}\right) .
$$

Using (25) - (29), $v^{a}=e_{4}^{a}$ and the components of Riemann tensor for the metric (2) (see appendix), in (24), we get

$$
\begin{gathered}
\frac{D^{2} \eta^{r}}{D \tau^{2}}=-\frac{3 e^{2}-2 m r}{r^{4}} \eta^{r}, \\
\frac{D^{2} \eta^{\theta}}{D \tau^{2}}=\frac{e^{2}-m r}{r^{4}} \eta^{\theta}, \\
\frac{D^{2} \eta^{\phi}}{D \tau^{2}}=\frac{e^{2}-m r}{r^{4}} \eta^{\phi},
\end{gathered}
$$

In order to write equation (30) - (32) in terms of ordinary derivative, we must evaluate the second covariant derivative derivative $\frac{D^{2}}{D \tau^{2}}$. Using $e_{4}^{a}=v^{a}$, equation(23) takes the form:

$$
\frac{D \eta^{\alpha}}{D \tau}=\frac{d \eta^{\alpha}}{d \tau}+\tilde{\Gamma}_{a b}^{\alpha} \eta^{b} v^{a}
$$

where

$$
\tilde{\Gamma}_{a b}^{\alpha}=e_{e}^{\alpha} e_{a}^{f} e_{b}^{g} \Gamma_{f g}^{e}
$$


Differentiating (33) covariantly, using the Christoffel components of metric (2) (see appendix), we can write (30) - (32) in the form

$$
\begin{gathered}
\frac{d^{2} \eta^{r}}{d \tau^{2}}=-\frac{3 e^{2}-2 m r}{r^{4}} \eta^{r}, \\
\frac{d^{2} \eta^{\theta}}{d \tau^{2}}=\frac{e^{2}-m r}{r^{4}} \eta^{\theta}, \\
\frac{d^{2} \eta^{\phi}}{d \tau^{2}}=\frac{e^{2}-m r}{r^{4}} \eta^{\phi},
\end{gathered}
$$

If the quantity $3 e^{2}-2 m r$ is positive (negative), then this indicates a tension or stretching (pressure or compression) in radial direction. Similarly, if $e^{2}-m r$ is positive (negative), this indicates a pressure or compression (tension or stretching) in the transversal directions.

\section{Conclusion}

The theory of differential spaces is used in gluing two Reissner-Nordström space-times $M_{1}$ and $M_{2}$ at their singularities .It is found that a light ray in the black hole of $M_{1}$ can go continuously through the black hole of $M_{2}$. The behavior of nearby timelike geodesics with zero angular momentum are investigated via the geodesic deviation equations. It is shown that there are a tension (or stretching) both in the radial and transversal direction according as the quantities $3 e^{2}-2 m r$ and $e^{2}-m r$ are positive(or negative)respectively.

\section{Appendix}

We use $\left(x^{1}, x^{2}, x^{3}, x^{4}\right)=(r, \theta, \phi, t)$ so that the non-vanishing Christoffel symbols of the second kind of the line element (1.1)are

$$
\begin{gathered}
\Gamma_{11}^{1}=\frac{e^{2}-m r}{r\left(r^{2}-2 m r+e^{2}\right)}, \Gamma_{12}^{2}=\frac{1}{r}, \\
\Gamma_{22}^{1}=-\frac{r^{2}-2 m r+e^{2}}{r}, \Gamma_{13}^{3}=\frac{1}{r}, \\
\Gamma_{33}^{1}=-\frac{\left(r^{2}-2 m r+e^{2}\right) \sin ^{2} \theta}{r}, \Gamma_{33}^{2}=-\sin \theta \cos \theta, \\
\Gamma_{44}^{1}=\frac{\left(r^{2}-2 m r+e^{2}\right)\left(\frac{m}{r^{2}}-\frac{e^{2}}{r^{3}}\right)}{r^{2}}, \Gamma_{23}^{3}=\cot \theta, \\
\Gamma_{14}^{4}=-\frac{r^{2}\left(-\frac{m}{r^{2}}+\frac{e^{2}}{r^{3}}\right)}{r^{2}-2 m r+e^{2}} .
\end{gathered}
$$


The non-zero Riemann tensor are:

$$
\begin{gathered}
R_{212}^{1}=\frac{e^{2}-m r}{\left.r^{2}\right)}, R_{313}^{1}=\frac{\left(e^{2}-m r\right) \sin ^{2} \theta}{r^{2}} \\
R_{414}^{1}=\frac{\left(r^{2}-2 m r+e^{2}\right)\left(3 e^{2}-2 m r\right)}{r^{6}}, R_{323}^{2}=\frac{\left(2 m r-e^{2}\right) \sin ^{2} \theta}{r^{2}}, \\
R_{424}^{2}=-\frac{\left(r^{2}-2 m r+e^{2}\right)\left(e^{2}-m r\right)}{r^{6}} \\
R_{434}^{3}=-\frac{\left(r^{2}-2 m r+e^{2}\right)\left(e^{2}-m r\right)}{r^{6}} .
\end{gathered}
$$

\section{References}

[1] Abdel-Megied M, Buchner K. and Gad R. M., (1996), "Topologie und Verklebung singulärer Raum-Zeiten". Proc. 4th Intern. Congr. Geometry, N. K. Artacutee miadis and N. K. Stephanidis ed., Thessaloniki , pp. 5768.

[2] Beigel A., and Buchner K., (1999), "d-spaces, singularities and the origin of charge". Proc. of the 4th Internat. Workshop on Differential Geometry, Brasov (Romania). Ed: Gh. Pitis and Gh. Munteanu pp. 46-55.

[3] Bossardt B., (1976), Comm. Math. Phys., 46, pp. 263-268.

[4] Buchner K., (1997), "Differential spaces and singularities of space-time". General Mathematics 5, pp. 53-66.

[5] Buchner K. and Büschel K., (1993). "Dynamical System on Differential Spaces", Proc. 23rd National Conf. on Geometry and Topology, ClujNapoca, pp. 30-37.

[6] Chandrasekhar, (1983). "The Mathematical Theory of Black Holes" (Oxford University Press, Cambridge, England).

[7] D'Inverno R., (1992), "Introducing Einstein's Relativity", Clarendon Press Oxford.

[8] Gad R. M., (1996), "some aspects of singularities in general relativity", Ph. D. Thesis, Minia University, Egypt.

[9] Geroch R., (1968), J. Math. Phys., 9, pp.450-468.

[10] Gerstner M., (1995), "d-Raume. Eine Verallgemeinerung der Differentialraume mittels Funktionsgarben", Dissertation, TU Munchen.

[11] Gerstner M. and Buchner K., (1996), "Differential Spaces Based on Local Functions". Analele Stiintifice ale Universitatii "Al. I. Cuza", Iasi, 42 (Suppl.) pp. 101-111.

[12] Gerstner M. and Buchner K., (1996), "The Topology of Differential Spaces". Analele Stiint. ale Univ. Al. I. Cuza, Iasi, 42 (Supliment), pp.101-111. 
[13] Gruszczak, J., Heller M., P., and Multarzynski, P., (1988). J. Math. Phys., 29 pp. 2576-2580.

[14] Gruszczak J., Heller M., and Multarzynski P., (1989), Foundations of Physics 19 pp. 607-618.

[15] Gruszczak J., Heller M. and Sasin W., (1992), Acta Cosmologica, 18 pp. 45-55.

[16] Hawking S.W. and Ellis G.F.R., (1973), "The Large Scale Structure of Space- Time", Cambridge Univ. press, Cambridge.

[17] Heller M., Multarzynski P. and Sasin W., (1989). Acta Cosmologica 16 pp. 53-85.

[18] Heller M. and Sasin W., (1994), Gen. Relat. Grav. 26, pp. 797-811.

[19] Heller M. and Sasin W., (1995), J. Math. Phys., 36, pp. 3644-3663.

[20] Lanczos K., (1924), Ann. Physik 74, pp. 518-540.

[21] Mostow M. A., (1979), J. Diff. Geom., 14, pp. 255-293.

[22] Sikorski R., (1971), Colloq. Mathem., 18, pp. 252-272., Colloq. Mathem., 24 (1971), pp. 46-79.

[23] Wald R.M., (1984), "General Relativity", Chicago and London. 